\title{
Automated Sperm Immobilization for Intracytoplasmic Sperm Injection
}

\author{
Clement Leung, Zhe Lu, Member, IEEE, Navid Esfandiari, Robert F. Casper, and Yu Sun*, Senior Member, IEEE
}

\begin{abstract}
Sperm immobilization is a requisite step in intracytoplasmic sperm injection (ICSI). Conventionally, sperm immobilization is performed manually, which entails long training hours and stringent skills. Manual sperm immobilization also has the limitation of low success rates and poor reproducibility due to human fatigue and skill variations across operators. This paper presents a system for fully automated sperm immobilization to eliminate limitations in manual operation. Integrating computer vision and motion control algorithms, the automated system is able to visually track a sperm and control a micropipette to immobilize the sperm. A robust sperm tail tracking algorithm is developed to locate the optimal position on the sperm tail for sperm immobilization. The system demonstrates: 1) an average sperm tail tracking error of $0.95 \mu \mathrm{m}$; 2) a sperm tail visual tracking success rate of $96 \%$; ) a sperm immobilization success rate of $88.2 \%$ (based on 1000 trials); and 4) a speed of 6-7 s per successful immobilization.
\end{abstract}

Index Terms-Computer vision, lab automation, micromanipulation, microrobotics, microscopy.

\section{INTRODUCTION}

$\mathbf{I}$ NTRACYTOPLASMIC sperm injection (ICSI) is a medical procedure that has enabled the in vitro fertilization of a single egg with a single sperm [1]. The procedure consists of three main steps: immobilizing a sperm, aspirating the sperm into a micropipette, and injecting the sperm into an egg [2]. Sperm immobilization must be performed before the insertion of the sperm into the egg to increase the chance of fertilization since sperm tail movement can cause damage to the intracellular structure of the egg [3]. Conventionally, ICSI has been performed manually by highly trained ICSI technicians/embryologists. Manual ICSI suffers from a long learning curve, varying success rates across operators, and poor performance consistency across clinics. The past decade witnessed significant efforts in automating

Manuscript received August 24, 2010; revised November 1, 2010 and November 26, 2010; accepted November 29, 2010. Date of publication December 13, 2010; date of current version March 18, 2011. C. Leung and Z. Lu share first authorship. This work was supported from the Natural Sciences and Engineering Research Council of Canada and Ontario Centres of Excellence. Asterisk indicates corresponding author.

C. Leung and Z. Lu are with the Advanced Micro and Nanosystems Laboratory, University of Toronto, Toronto M5S 3G8, Canada (e-mail: clement.leung@utoronto.ca; zhe.lu@utoronto.ca).

*Y. Sun is with the Advanced Micro and Nanosystems Laboratory, University of Toronto, Toronto M5S 3G8, Canada (e-mail: sun@mie.utoronto.ca).

N. Esfandiari is with the Assisted Reproduction Technologies Laboratories, Toronto Centre for Advanced Reproductive Technology, Toronto, ON M5S 2X9, Canada (e-mail: nesfand@yahoo.com).

R. F. Casper is with the Department of Obstetrics and Gynaecology, University of Toronto, Toronto M5S 3G8, Canada (e-mail: casper@lunenfeld.ca).

Color versions of one or more of the figures in this paper are available online at http://ieeexplore.ieee.org.

Digital Object Identifier 10.1109/TBME.2010.2098875 the injection of cells [4]-[7]. However, the automation of sperm manipulation has not yet been attempted, which is an important endeavor for ultimately standardizing clinical ICSI.

Sperm immobilization requires a micropipette to press (tap) the sperm tail against a surface (e.g., the bottom of a Petri dish) [3]. It is a challenging procedure that has stringent skill requirements. Due to the fast movement of a healthy sperm $(\geq 25 \mu \mathrm{m} / \mathrm{s}$ ) [8], a sperm can move out of the field of view of a microscope quickly. The operator needs to carefully monitor the motion of the sperm and manually move the microscope stage to keep the sperm within the field of view, while simultaneously attempting to tap the sperm tail for immobilization.

For immobilizing a sperm, consistently tapping the midpoint of the sperm tail is preferred to prevent damage to the sperm head [3]. Due to the small size of the sperm tail $(\leq 1 \mu \mathrm{m}$ in thickness), the visibility of the sperm tail is rather low under optical microscopy conditions (e.g., bright field microscopy, Hoffman modulation contrast, and differential interference contrast), making the identification and visual tracking of the midpoint of a moving sperm tail arduous for both novice and proficient operators.

To create a system for automated sperm immobilization, sperm head and tail tracking algorithms are essential for: 1) visually tracking the spatial location of the sperm; 2) servoing the microscope stage to move the sperm to the center of the field of view; and 3) averaging the tracked head and tail positions to locate the midpoint of the sperm tail for robotic immobilization. Several algorithms have been developed in the field of computer-assisted sperm analysis (CASA) to track sperm trajectories, measure sperm velocity, and evaluate sperm energetics [9]-[11]. Prior work for estimating the direction of a sperm tail used the minor and major axes of the sperm head's morphology [12]. The sperm head, however, has a wide variety of shapes [8]. Thus, the approach does not always provide the correct direction of the sperm tail. Despite the considerable progress made in CASA [13], automated detection and tracking of a sperm tail, which is essential for sperm immobilization, has not been explored.

The low contrast of the sperm tail under optical microscopy is a major reason why sperm tail tracking is challenging. Tracking low-contrast objects has long been a topic of interest in computer vision. Since edge information is often lost or cannot be extracted from low-contrast objects in images, Chan and Vese developed an active contour-based object detection algorithm that does not require edge information [14]. The method has been demonstrated to form contours around objects of low contrast. However, the method requires numerous iterations before converging to a solution, rendering the algorithm unsuitable for 
real-time applications, such as sperm tail tracking. The Kalman filter has been shown to be capable of tracking low-contrast objects [15]. In fact, the Kalman filter is suitable for tracking objects that exhibit linear and Gaussian temporal dynamics [16]. However, the motion of a sperm tail is sporadic, multimodal, and hence, non-Gaussian, making the Kalman filter unsuitable for sperm tail tracking. Tsai et al. developed an independent component analysis-based filter for detecting low-contrast defects on LCD glasses in uniform surface images [17]. Their approach has been applied to detecting inanimate low-contrast objects. However, it does not take into account the temporal dynamic changes that are prevalent in moving objects, such as motile sperm.

This paper presents an automated system that is capable of performing sperm immobilization with a high success rate. Visual tracking and servoing algorithms are integrated into the system for tracking the sperm position, finding the midpoint of the sperm tail, and controlling multiple motion control devices for sperm immobilization. The sperm tail tracking algorithm is robust to the low-contrast appearance and the nonlinear fast movement of the sperm tail. The success rate of sperm tail tracking was evaluated on 100 sperm. The success rate of automated sperm immobilization was quantified via 1000 trials.

\section{SYSTEM DESIGN}

\section{A. System Architecture}

As shown in Fig. 1, the automated sperm immobilization system consists of a standard inverted microscope (bright field imaging, Nikon TE2000-S) and a motorized $X-Y$ translational stage (ProScan, Prior Scientific, Inc.). The stage has a travel range of $75 \mathrm{~mm}$ along both axes with a resolution of $0.01 \mu \mathrm{m}$, a maximum speed of $5 \mathrm{~mm} / \mathrm{s}$, and a repeatability of $\pm 1 \mu \mathrm{m}$. A $20 \times$ objective with a numerical aperture of 0.45 is used (CFI Plan Fluor ELWD, Nikon). A CMOS camera (601f, Basler; resolution: $640 \times 480$ ) is connected to the microscope for providing visual feedback with the following parameters: shutter 1400 , gain 80 , and brightness 1600 . A straight ICSI micropipette (outer diameter: $6 \mu \mathrm{m}$ ) tilted at $30^{\circ}$ is attached to a 3-DOF motorized micromanipulator (MP285, Sutter, Inc.) that has a travel range of $25 \mathrm{~mm}$ and a $0.04 \mu \mathrm{m}$ positioning resolution along each axis.

\section{B. Overall Operation Sequence}

The system starts by performing vision-based contact detection [18] to determine the relative vertical depth positions between the micropipette tip and surface of the Petri dish [see Fig. 2(a)]. The micropipette is then positioned at $25 \mu \mathrm{m}$ above the bottom of the Petri dish and $100 \mu \mathrm{m}$ to the left of the center of the field of view [see Fig. 2(b)]. Note that contact detection only needs to be performed once at the start of the system. A human operator then selects a sperm of interest by computer mouse clicking on or in proximity to the head of the sperm [see Fig. 2(b)]. This step permits the human operator to select a desired sperm based on sperm morphology and motility, thus exercising his/her expert knowledge. The system then starts to track the sperm head and tail [see Fig. 2(c)]. The located sperm

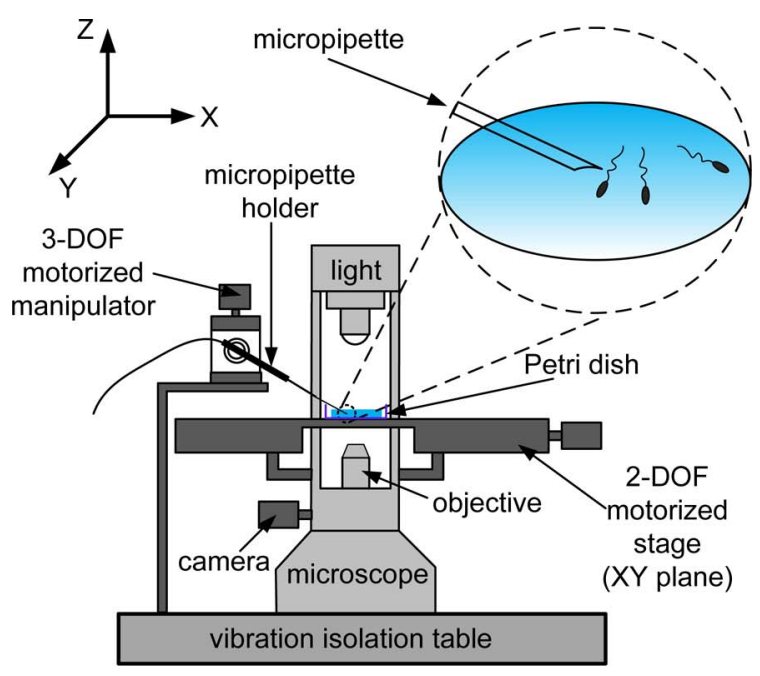

(a)

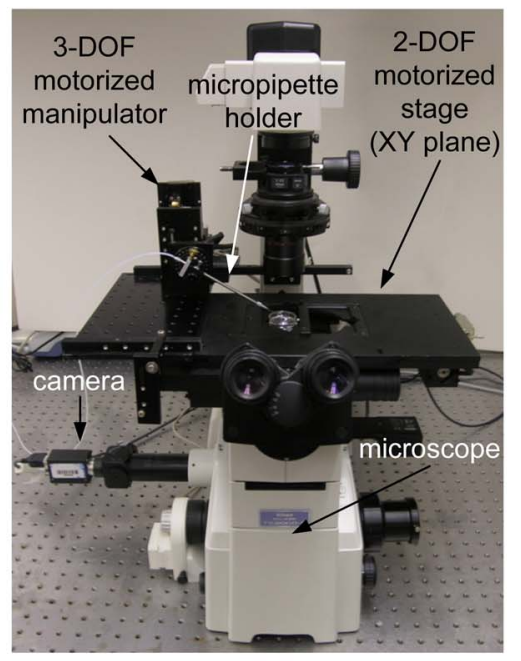

(b)

Fig. 1. Automated sperm immobilization system. (a) Schematic illustration. (b) Picture of the system.

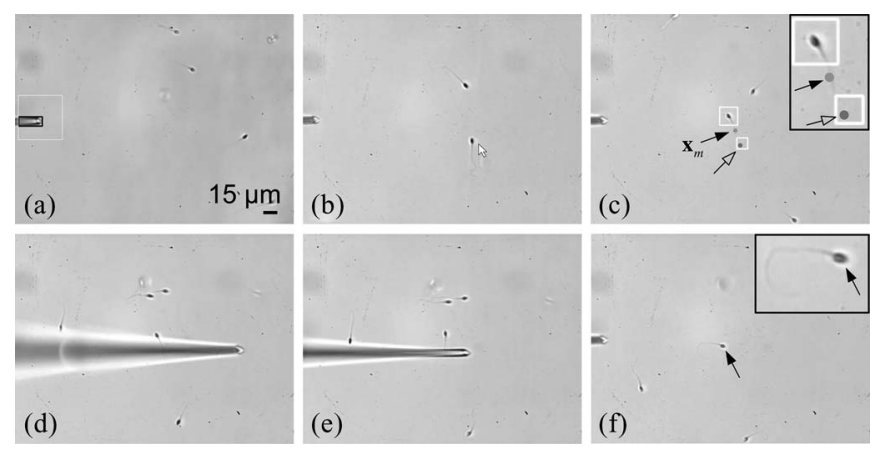

Fig. 2. Sperm immobilization operation sequence. (a) Contact detection is executed to compute the relative depths between the Petri dish and micropipette. (b) Human operator selects a sperm of interest by computer mouse clicking on the sperm head. (c) Sperm head and tail tracking is performed. The sperm is maintained at the center of the field of view by visually servoing the $X-Y$ stage. The black arrow points to the calculated tail midpoint position $\mathbf{x}_{m}$ and the hollow arrow points to the calculated tail position. (d)-(e) show the sperm tapping process. (f) Sperm is immobilized. 


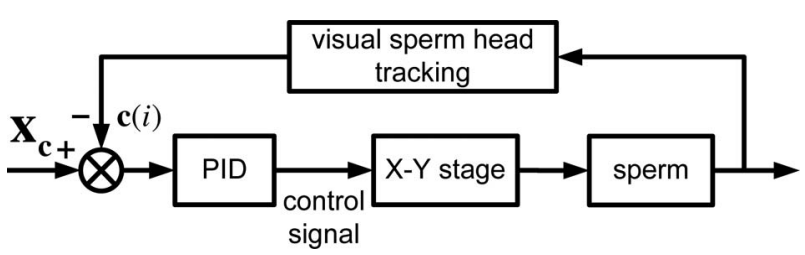

Fig. 3. Control diagram for the visual servoing of the $X-Y$ stage.

head position enables the $X-Y$ stage to move the sperm of interest to the center region of the field of view. The midpoint of the sperm tail $\mathbf{x}_{m}$ is found by averaging the tracked head and tail position. The micromanipulator undergoes a sequence of motions to tap the midpoint of the sperm tail against the bottom of the Petri dish to immobilize the sperm [see Fig. 2(d) and (e)]. Finally, the micropipette is brought back to its original resting position of $25 \mu \mathrm{m}$ above the bottom of the Petri dish and $100 \mu \mathrm{m}$ to the left of the center of the field of view, ready for the next sperm immobilization operation [see Fig. 2(f)].

\section{SPERM TRACKING}

\section{A. Sperm Tracking Overview}

Our sperm tracking algorithm tracks two regions of the sperm: the sperm head position and the sperm tail position. The algorithm has three steps. Step-1 tracks the sperm head to provide visual feedback of the sperm position. In Step-2, the sperm tail region of interest (STROI) is extracted. STROI extraction is an extrapolation process that calculates the region in which the sperm tail is located by using information from Step-1. The STROI aims to capture the tail tip region of the sperm. Once the STROI is found, the maximum intensity region (MIR) algorithm is used to locate a point on the sperm tail within the STROI. Finally, the midpoint of the sperm tail $\mathbf{x}_{m}$ is calculated by averaging the tracked head and tail position.

\section{B. Sperm Head Tracking and Visual Servoing}

The sperm head is distinctive and hence is used for tracking the position of a sperm. With the sperm head position, $\mathbf{c}(i)=\left(c_{x}(i), c_{y}(i)\right)$, the spatial distance from the sperm head $\mathbf{c}(i)$ to the center of the field of view $\mathbf{X}_{c}$ is determined. The spatial distance is input into a PID controller, which is used to visually servo the $X-Y$ stage to keep the sperm at the center region of the field of view [see Fig. 2(c)]. Starting values of PID gains were obtained using standard methods, and their final values were determined through experimental trials. Fig. 3 shows the control diagram for the visual servoing process. The accuracy tolerance to the center of the field of view is 1 pixel or $1.48 \mu \mathrm{m}$. It can take several seconds for a sperm to reach within the required orientation before immobilization can be conducted (Section IV-A). Thus, the distance calculation and servoing of the $X-Y$ stage is performed for every frame of image $(30 \mathrm{~Hz})$ to maintain the sperm at the center region of the field of view. Keeping the sperm at the center of the image increases the robustness of the overall sperm tracking algorithm as it prevents the sperm from moving out of the field of view.

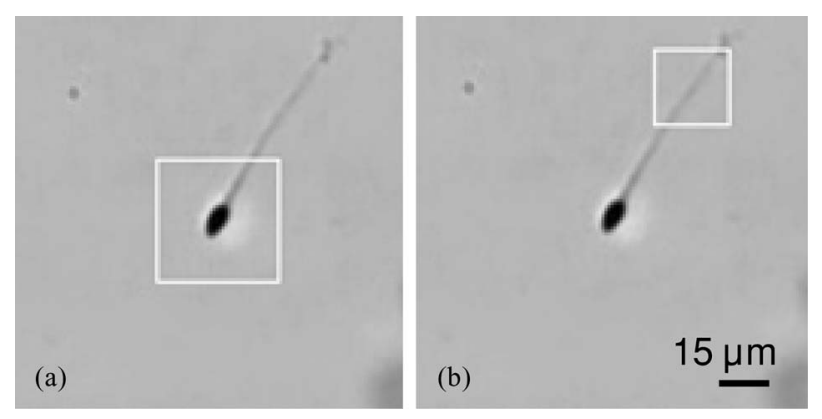

Fig. 4. (a) Example of the SHROI shown by the white box around the sperm head. (b) Example of the STROI shown by the white box around the sperm tail.

The sperm head tracking process is initiated by a human operator who selects a desired sperm head to track via mouse click on or within proximity to the sperm head [see Fig. 2(b)]. The system obtains a $40 \times 40$ sperm head region of interest (SHROI) from the current frame $i$. The SHROI image's center is initially at the mouse clicked position. The SHROI image is binarized by applying Otsu's adaptive thresholding algorithm [19]. A morphological close operation is performed to remove noise and small particles that may be present in the SHROI image. The result is a binary SHROI image, where the pixel value is 1 for an object pixel and 0 otherwise. The contour of the sperm head in the SHROI image is computed. The sperm head position $\mathbf{c}(i)$ is found by calculating the moment of the contour. The SHROI is then updated to be a $40 \times 40$ region of interest centered at the sperm head's centroid, as shown in Fig. 4(a). For subsequent frames, a similar process is executed to track the sperm head position. However, instead of a mouse click to initiate the process, the SHROI of the previous frame is used by the system as the initial $40 \times 40$ SHROI.

There may be instances in which foreign particles or other sperm enter the SHROI. The sperm head tracking algorithm must be able to differentiate between the sperm of interest and interfering sperm or foreign objects that enter the SHROI. The swimming direction vector of the sperm of interest is used as a unique identifier to discriminate the sperm of interest from other sperm or particles. In the situation where only one sperm is present in the SHROI, the sperm's current direction vector $\mathbf{v}(i)$ in the current frame, represented by (1), is found by subtracting the sperm centroid position in the previous frame $\mathbf{c}(i-1)$ from the sperm centroid position in the current frame $\mathbf{c}(i)$

$$
\mathbf{v}(i)=\mathbf{c}(i)-\mathbf{c}(i-1) .
$$

When more than one sperm or object is present in the SHROI, (1) is extended to

$$
\mathbf{v}(i, s)=\mathbf{c}(i, s)-\mathbf{c}\left(i-1, s_{\text {prev }}\right)
$$

where $s$ represents each sperm in the SHROI, and $s_{\text {prev }}$ is the sperm of interest in the previous frame. The candidate sperm $s$ that produces the minimum Euclidean distance value is considered the sperm of interest $s_{\text {soi }}$

$$
s_{\text {soi }}=\min _{s \in[1, N]}\left\{\left\|\mathbf{v}(i, s)-\mathbf{v}\left(i-1, s_{\text {prev }}\right)\right\|\right\}
$$


where $N$ is the total number of sperm and objects inside the SHROI. Specifically, the nearest neighbor approach is applied to determine the sperm of interest $s_{\text {soi }}$ at instance $i$, with the knowledge of the sperm of interest $s_{\text {prev }}$ at instance $i-1$. The $s_{\text {soi }}$ is updated for every frame using this nearest neighbor computation.

\section{Sperm Tail Tracking}

With the sperm head position found, the STROI in which the tail is located can be computed. An example of the STROI is shown in Fig. 4(b). In the ICSI procedure, healthy energetic sperm with strong movement are desired. Hence, our tracking algorithm is only concerned with sperm that are in motion. The average unit direction vector of the sperm movement and the sperm head position are used to find the STROI. The unit average direction vector is used instead of the direction vector described by (3), because the sperm may exhibit abrupt local changes in movement direction between two consecutive frames. By averaging the direction vectors of the sperm across 30 frames, abrupt changes in the sperm direction between frames are mitigated. Each spatial component of the average direction vector $\overline{\mathbf{v}}(i)$ for a given frame $i$ is found by

$$
\overline{\mathbf{v}}(i)=\frac{1}{30} \sum_{k=0}^{29}\left(c_{r}(i-k)-c_{r}(i-k-1)+\varepsilon_{r}(i-k)\right)
$$

where $r$ represents the spatial coordinate values $x$ and $y$, and $\varepsilon_{r}$ denotes displacements produced by the stage. The average direction vector is calculated to be the sperm's velocity in pixels/s. Each spatial component of the unit average vector $\hat{v}_{r}(i)$ can then be calculated to be

$$
\hat{v}_{r}(i)=\frac{\bar{v}_{r}(i)}{\|\overline{\mathbf{v}}(i)\|} .
$$

The STROI's center position $t_{r}(i)$ is found by adding a scaled value of the direction vector to the sperm head's centroid

$$
t_{r}(i)=c_{r}(i)+a \cdot \hat{v}_{r}(i)
$$

where $a$ is a scalar value. Studies have shown that the average human sperm length is approximately $50 \mu \mathrm{m}$ [8]. Under $20 \times$ magnification, this value converts to a length of approximately 120 pixels. Experimentally, we found that a value [70, 90] is appropriate for $a$, as the value compensates for the sperm tail's constant contraction and bending. The STROI is obtained by taking a $25 \times 25 \mathrm{ROI}$ centered at the $t_{r}(i)$ position. A $25 \times 25 \mathrm{STROI}$ provides a sufficient tail search area that takes into account a range of different sperm tail lengths and the tail's transversal displacement.

After finding the STROI, the algorithm verifies that a tail is present in the STROI. The fundamental feature of flicker is extracted by taking the absolute difference between six consecutive inverted grayscale image frames [20]

$$
f(i)=\sum_{k=0}^{5}|I(i-k)-I(i-k-1)|
$$

where $f(i)$ is the flicker image extracted at frame $i$, and $I$ represents the grayscale images containing the sperm of interest

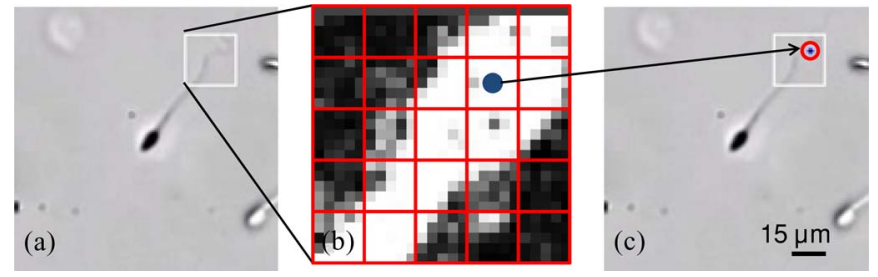

Fig. 5. Steps of the MIR algorithm. (a) STROI is found. (b) $5 \times 5$ window (represented by the red boxes) is used to scan and find the $5 \times 5$ section with the highest intensity sum in the flicker image. The center point (blue dot) of the $5 \times 5$ window with the highest intensity sum is considered the tail location. (c) Frame displaying the MIR's estimation of a point on the sperm tail.

at frames $i$ to frame $i-5$. Each pixel in the flicker image is squared to enhance the pixel values of areas in which the tail is present. The pixel sum in the STROI of the $f(i)$ image is used as a measurement to determine the presence of a sperm tail. If the pixel sum is above a specific threshold $(\beta=8000)$, a tail is considered to be present. The threshold value $\beta$ was found experimentally by comparing the pixel sum values of STROI images in which a tail exists against cases where no tail exists. Since the flicker image excludes uniform background information, this $\beta$ value can be applied to different microscopic brightness conditions. However, $\beta$ may need to be adjusted to compensate for variations in noise floor conditions across different microscope cameras.

An example of the flicker image is shown in Fig. 5(b). If the pixel sum is below the threshold value $\beta$, no tail can be found and the human operator will be informed via a prompt message that the tail has been lost. This situation can happen if the sperm of interest becomes out of focus, resulting in the tail disappearing from the field of view.

Using the center position of the STROI is not always sufficient for accurately locating the sperm tail midpoint due to variations in sperm tail lengths. Hence, once the sperm tail is confirmed to exist within the STROI, the MIR algorithm is used to detect a point on the sperm tail and to compute the sperm tail midpoint position. The motivation behind this algorithm is that an individual frame may not show the sperm tail due to the tail's low contrast and fast movement. However, by extracting the flicker feature of the sperm tail [as shown in Fig. 5(b)], the position of the sperm tail can be prominently seen. This approach overcomes challenges that arise from the low-contrast image of the sperm tail in a single frame. Compared with the optical flow method [21], the MIR algorithm experimentally produced a higher accuracy in locating a point on the sperm tail.

The MIR algorithm first finds the location of maximum intensity within the $25 \times 25$ STROI of the flicker image. This is accomplished by evaluating the sum of the intensity values inside a 5 pixel by 5 pixel window at a spatial sampling interval of 5 pixels in both the $x$ and $y$ coordinates of the STROI flicker image. Experimentally, we found that $5 \times 5$ was an appropriate sampling window for accurately locating a position on the sperm tail. A larger window provides less accurate results while a smaller window size is more susceptible to noise. The center position of the $5 \times 5$ window with the largest intensity value is considered the tail location (i.e., a point on the sperm tail). Fig. 5 


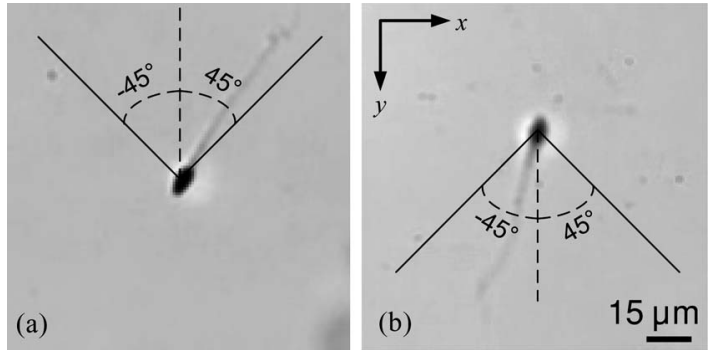

Fig. 6. Sperm tail orientation criteria.

provides an illustrative example of the MIR algorithm's tracking process.

\section{SPERM IMMOBILIZATION}

After the system obtains sperm head and tail positions from visual tracking, the system determines whether the sperm is within the ideal orientation and depth requirements for immobilization. Both criteria need to be satisfied before the system controls the micropipette to immobilize the sperm.

\section{A. Criteria}

Two conditions must be met for the sperm tail tapping step to execute: 1) the sperm tail needs to be in an appropriate orientation and 2) the sperm cannot exceed a depth of $25 \mu \mathrm{m}$ above the bottom of the Petri dish. The system continuously performs sperm tracking [see Fig. 2(c)] until both criteria are met. Our software permits the human operator to cancel sperm tracking and to select a different sperm if the two aforementioned criteria are not met within a reasonable amount of time.

1) Sperm Tail Orientation: In our system, the micropipette is on the left-hand side of the field of view [see Fig. 2(a)]. For successful sperm immobilization, the sperm tail's orientation needs to be within $-45^{\circ}$ to $45^{\circ}$ with respect to the $y$-axis, as shown in Fig. 6. Experiments prove that it is difficult for the micropipette to tap sperm tails with orientations outside this range. Thus, the system is configured to perform sperm immobilization only when the sperm tail is within the aforementioned range for a duration of at least $1 \mathrm{~s}$. The system waits for the sperm to move within the required orientation for performing the immobilization task. The use of a rotational stage to orient a sperm can possibly reduce this waiting time; however, one must note that the sperm can move out of the field of view because of the coupled translational motion from rotation. The sperm tail's orientation $\theta$ with respect to the $y$-axis is determined as

$$
\theta=\arctan \frac{\hat{v}_{x}(i)}{\hat{v}_{y}(i)} .
$$

2) Sperm Depth: To immobilize a sperm, the micropipette tip needs to tap the sperm tail against the surface of the Petri dish. The vertical position of a sperm exceeding a certain depth (e.g., $25 \mu \mathrm{m}$ ) above the Petri dish surface often makes immobilization fail since the micropipette is unable to press the tail against the Petri dish at these depths, although the system is still able to track the sperm. In addition, at these depths, the micropipette moving

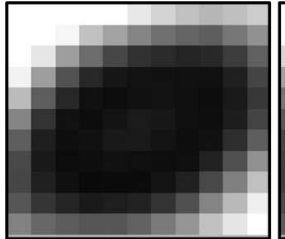

(a)

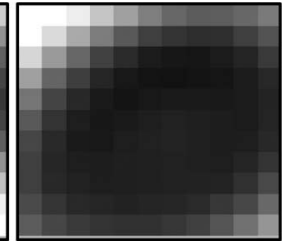

(b)

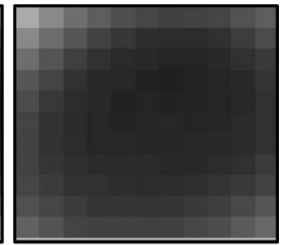

(c)
Fig. 7. Sperm head image at different focus depths. (a) $0 \mu \mathrm{m}$ (dish bottom). (b) $15 \mu \mathrm{m}$. (c) $25 \mu \mathrm{m}$.

TABLE I

SPERM TAIL TAPPING SEQUenCE

\begin{tabular}{llll}
\hline step & micropipette & visual tracking & $\mathrm{X}-\mathrm{Y}$ stage \\
\hline 1 & moved near $\mathbf{x}_{m}$ & enabled & servoing \\
2 & position maintained & disabled & constant velocity \\
3 & tapping & disabled & constant velocity \\
4 & returned to resting position & disabled & stopped \\
\hline
\end{tabular}

for sperm immobilization often causes strong disturbances to the medium and displaces the sperm from its original position. Accordingly, the system is programmed not to perform sperm tail tapping unless the sperm depth is between 0 and $25 \mu \mathrm{m}$ above the Petri dish surface.

The system computes the focus measure by using the normalized variance [22] of a $10 \times 10 \mathrm{ROI}$ image of the sperm head (see Fig. 7). The normalized variance, NormVar is calculated as follows:

$$
\text { NormVar }=\frac{1}{H W \bar{I}} \sum_{\text {height }} \sum_{\text {width }}(I(x, y)-\bar{I})^{2}
$$

where $I$ is the ROI image, $H$ is the height of the ROI image, $W$ is the width of the ROI image, and $\bar{I}$ is the expected pixel value of the ROI image.

The microscope is focused on the bottom level of the Petri dish in our system. A sperm at this level exhibits the highest normalized variance values since this is the optimal point of focus. In contrast, a sperm that is above Petri dish surface, as shown in Fig. 7, has lower normalized variance values due to the blurring of the sperm head. Experiments demonstrate that all sperm at a depth greater than $25 \mu \mathrm{m}$ have normalized variance values, NormVar less than 8 for the optical conditions described in Section II-A. This NormVar threshold value needs to be recalibrated when significantly different imaging conditions are used.

\section{B. Sperm Tail Tapping}

When both criteria described in Section IV-A are satisfied, the system executes sperm tail tapping. The micromanipulator is programmed to execute a sequence of motions to tap the calculated sperm tail midpoint $\mathbf{x}_{m}$ against the bottom of the Petri dish. Automated sperm tapping consists of four main steps.

Table I shows the states of the micropipette, sperm tracking, and $X-Y$ stage for each step of the sperm tail tapping sequence. The duration of the sperm tail tapping sequence is within $3 \mathrm{~s}$. 
TABLE II

AVERAGE EUCLIDEAN DistANCE ERROR

\begin{tabular}{lllllll}
\hline & V1 & V2 & V3 & V4 & V5 & Average \\
Duration & $3.83 \mathrm{~s}$ & $6.67 \mathrm{~s}$ & $3.83 \mathrm{~s}$ & $4.60 \mathrm{~s}$ & $3.87 \mathrm{~s}$ & \\
\hline MIR (pixels) & 1.16 & 2.73 & 1.83 & 2.02 & 1.98 & 1.95 \\
\hline
\end{tabular}

In Step-1, the micromanipulator positions the micropipette $30 \mu \mathrm{m}$ horizontally to the left of the $\mathbf{x}_{m}$ position. In Step-2, sperm head and tail tracking are disabled to prevent tracking errors due to the occlusion of the sperm by the micropipette. After visual tracking is disabled, the $X-Y$ stage continues to move at the last PID computed velocity. In Step-3, the micromanipulator moves the micropipette to a position $100 \mu \mathrm{m}$ horizontally to the right of $\mathbf{x}_{m}$. The micromanipulator moves at a relatively low speed of $200 \mu \mathrm{m} / \mathrm{s}$ to avoid creating disturbances in the medium that can displace the sperm. The micromanipulator's speed is then increased to $6000 \mu \mathrm{m} / \mathrm{s}$, while simultaneously lowering the micropipette vertically by $60 \mu \mathrm{m}$ [see Fig. 2(d)]. The act of displacing the micropipette by a vertical value that exceeds its vertical height $(25 \mu \mathrm{m})$, coupled with the large increase in micromanipulator speed, results in the bending of the micropipette tip against the bottom of the Petri dish. The bending causes the micropipette to press on the sperm tail. The sequence continues by moving the micropipette at a speed of $6000 \mu \mathrm{m} / \mathrm{s}$ horizontally while pressing against the sperm tail [see Fig. 2(e)] until the micropipette reaches a position of $60 \mu \mathrm{m}$ to the left of $\mathbf{x}_{m}$. The rapid dragging motion of the micropipette against the sperm tail in Step-3 ensures the immobilization of the sperm. Lastly, in Step-4, the $X-Y$ stage's velocity is set to 0 , and the micropipette is moved back to its original resting position [see Fig. 2(b)].

\section{EXPERIMENTAL RESUlTS AND DisCUSSION}

For all experiments, human sperm were placed in a Petri dish containing a viscous culture medium (SpermCatch, NidaCon International). SpermCatch is commonly used in clinical ICSI to create a fluid environment that mitigates adherence of the sperm to the dish or the micropipette, assists sperm aspiration, and helps slow down sperm motion [23]. Mineral oil was used to fully encapsulate the culture medium to prevent the culture medium from evaporation.

\section{A. Sperm Tail Tracking Accuracy}

Five videos (V1-V5 in Table II) of moving sperm captured under the optical conditions described in Section II-A were used to evaluate the performance of the MIR sperm tail tracking algorithm. These videos were recorded at 30 frames/s. During video capturing, the sperm were allowed to swim out of the field of view.

The performance of the tail tracking algorithm was evaluated by calculating the Euclidean distance error between the algorithm computed tail location and a reference position. The reference position is the location (carefully input by a user for each frame of a video via mouse click) on the sperm tail that produces the minimum Euclidean distance between the sperm tail and the algorithm computed sperm tail location. An exam-

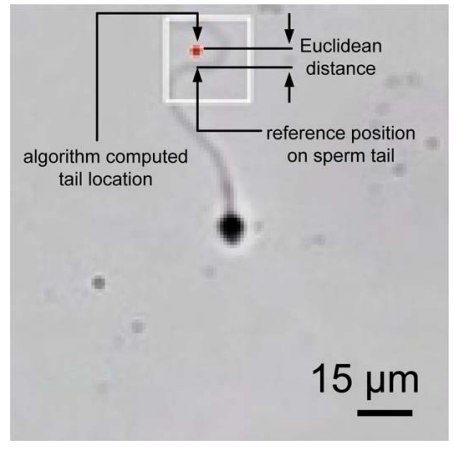

Fig. 8. Illustration of the Euclidean distance measured between an algorithm's calculated tail location and the reference position.

ple of the Euclidean distance measurement is shown in Fig. 8. The Euclidean distances are computed for each frame of each video.

Table II summarizes the average Euclidean distances for each video. The MIR sperm tail tracking algorithm is capable of accurately tracking the sperm tail's location. The MIR algorithm has an overall average Euclidean distance error of 1.95 pixels $(0.95 \mu \mathrm{m})$. As discussed in Section III-C, the MIR algorithm uses the current frame and the previous five frames to extract the flicker feature. Thus, the previous frames have an influence on the region of the STROI with the highest intensity value. As a result, the MIR algorithm's estimated tail location in certain cases is in proximity to the sperm tail, but not exactly on the sperm tail, contributing to the Euclidean distance error of the MIR algorithm. Furthermore, foreign objects such as debris or other sperm entering the STROI can cause the MIR algorithm's calculated tail position to stray away from the actual tail position. This situation occurred in Video 2, resulting in a slightly higher average Euclidean distance ( 2.73 pixels).

\section{B. Sperm Tail Tracking Success Rate}

The system performed 100 trials to quantify the sperm tail tracking success rate. Sperm tail tracking success rate is defined as the ability of the algorithm to follow the sperm tail from the moment when the sperm of interest is selected to when tracking is disabled during the micropipette sperm tail tapping step.

The system achieved a sperm tail tracking success rate of $96 \%$. Sperm tail tracking failed when the sperm contacted the thick dark boundary between the culture medium and the exterior. In this region, the sperm head is occluded by the dark boundary, causing sperm head tracking to fail and in consequence, the failure of sperm tail tracking. The sperm may also enter dark areas of the medium (e.g., air bubbles or large foreign particles), which also caused tracking to fail. This latter type of failure can be reduced by manually eliminating bubbles in the culture medium and by ensuring that the culture medium has a low level of contaminants during sample preparation. Based on these 100 trials and the subsequent 1000 trials for quantifying sperm immobilization success rate, where sperm samples were diluted as per clinical ICSI routines, we observed that potential failure cases such as a sperm colliding with another sperm, two 
TABLE III

AUTOMATED SPERM IMMOBILIZATION RESULTS

\begin{tabular}{|c|c|c|c|c|c|c|c|}
\hline Avg. sperm speed $\bar{u}(\mu \mathrm{m} / \mathrm{sec})$ & Num. immobilized / Num. trials & (a) & (b) & (c) & (d) & (e) & $\%$ Immobilized \\
\hline $20<\bar{u} \leq 30$ & $66 / 81$ & 1 & 2 & 2 & 2 & 8 & 81.5 \\
\hline $13<\bar{u} \leq 20$ & $301 / 345$ & 3 & 11 & 4 & 17 & 9 & 87.5 \\
\hline $8<\bar{u} \leq 13$ & $261 / 315$ & 1 & 15 & 18 & 17 & 3 & 82.9 \\
\hline $3<\bar{u} \leq 8$ & $189 / 191$ & 0 & 0 & 1 & 1 & 0 & 98.5 \\
\hline $0<\bar{u} \leq 3$ & $65 / 68$ & 2 & 1 & 0 & 0 & 0 & 95.6 \\
\hline Total & $882 / 1000$ & 7 & 29 & 25 & 37 & 20 & 88.2 \\
\hline
\end{tabular}

sperm moving in parallel within the SHROI, or a sperm moving in the $Z$-direction with no $X$ or $Y$ component, occurred extremely rarely, if at all.

\section{Sperm Immobilization Success Rate}

Trials on 1000 sperm were performed with the automated system to evaluate the system's efficacy for sperm immobilization. The average successful immobilization time was determined to be 6-7 s. Overall, the system achieved an immobilization success rate of $88.2 \%$ (882 out of 1000 sperm were successfully immobilized). Details are summarized in Table III.

The sperm were categorized into five groups based on their average speeds $(\mu \mathrm{m} / \mathrm{s})$. A sperm's average speed, $\bar{u}$ is calculated by averaging the Euclidean norm values $\|\overline{\mathbf{v}}(i)\|$ over the duration of sperm immobilization and then converting the result from pixels/s to $\mu \mathrm{m} / \mathrm{s}$. In Table III, columns (a)-(e) summarize five different causes of immobilization failure.

Failure case (a) happened when the micropipette disturbed the culture medium, which in turn displaced the sperm from its original position, causing the micropipette to miss the sperm tail. Case (a) accounted for $0.7 \%$ of the trials and tended to happen when the micropipette was close to the boundary between the culture medium and the surrounding mineral oil. Failure case (b) accounted for $2.9 \%$ of the trial results. Case (b) occurred when the sperm moved to a depth above $25 \mu \mathrm{m}$ and maintained a depth above $25 \mu \mathrm{m}$ for more than $30 \mathrm{~s}$ of tracking. In case (b), sperm tapping was not executed because of system configuration described in Section IV-A2.

Failure case (c) happened when the sperm tail did not stay within the required orientation (described in Section IV-A1) for more than $30 \mathrm{~s}$ of tracking. Thus, the system did not perform sperm tail tapping. Case (c) accounted for $2.5 \%$ of the trials. Case (d) (3.7\% of the trials) occurred when the sperm tail orientation changed to an orientation outside of the required orientation range during the sperm tapping step. This failure case happened most often for sperm that moved in a circular path. In a small number of cases $(2 \%)$ represented by (e), the sperm increased its speed during the sperm tail tapping step. Because sperm tracking is disabled in the sperm tapping step, the $X-Y$ stage is servoed at the last PID computed velocity. Thus, the constant velocity of the $X-Y$ stage is not capable of compensating for the increase in sperm speed, resulting in the micropipette either missing the sperm tail, or hitting the very tip of the sperm tail, which often is not sufficient for immobilizing the sperm due to the extreme thinness of the sperm tail tip.

Overall, the results show that each failure case accounts for only a small number of the trials. When sperm immobilization fails, the human operator can readily select the same sperm to attempt to immobilize it, or select a different sperm for tracking and immobilization.

\section{CONCLUSION}

This paper reported on a system for automated sperm immobilization. A visual tracking algorithm was developed to track a sperm's head and tail and locate the midpoint on the sperm tail for automated immobilization. The tracking algorithm was capable of tracking the sperm tail with an average error of $0.95 \mu \mathrm{m}$ and a high success rate of $96 \%$. Motion control algorithms were integrated into the system to control the micropipette for tapping the sperm tail to achieve immobilization. Experiments on 1000 sperm demonstrated that the system was able to perform automated sperm immobilization at a speed of 6-7 s per immobilization and with a success rate of $88.2 \%$.

\section{REFERENCES}

[1] G. Palermo, H. Joris, P. Devroey, and A. C. Van Steirteghem, "Pregnancies after intracytoplasmic injection of single spermatozoon into an oocyte," Lancet, vol. 340, no. 8810, pp. 17-18, 1992.

[2] P. Vanderzwalmen, G. Bertin, B. Lejeune, M. Nijs, B. Vandamme, and R. Schoysman, "Two essential steps for a successful intracytoplasmic sperm injection: Injection of immobilized spermatozoa after rupture of the oolema," Hum. Reprod., vol. 11, no. 3, pp. 540-457, 1996.

[3] G. D. Palermo, P. N. Schlegel, L. T. Colombero, N. Zaninovic, F. Moy, and Z. Rosenwaks, "Aggressive sperm immobilization prior to intracytoplasmic sperm injection with immature spermatozoa improves fertilization and pregnancy rates," Hum. Reprod., vol. 11, no. 5, pp. 1023-1029, 1996.

[4] Y. Sun and B. J. Nelson, "Biological cell injection using an autonomous microrobotic system,” Int. J. Robot. Res., vol. 21, no. 10-11, pp. 861-868, 2002.

[5] X. Y. Liu and Y. Sun, "Automated mouse embryo injection moves toward practical use," in Proc. IEEE Int. Conf. Robot. Autom., 2009, pp. 526-531.

[6] W. H. Wang, X. Y. Liu, and Y. Sun, "High-throughput automated injection of individual biological cells," IEEE Trans. Autom. Sci. Eng., vol. 6, no. 2, pp. 209-219, Apr. 2009.

[7] X. Y. Liu and Y. Sun, "Microfabricated glass devices for rapid single cell immobilization in mouse zygote microinjection," Biomed. Microdevices, vol. 11, no. 6, pp. 1169-1174, 2009.

[8] World Health Organization, WHO Laboratory Manual for the Examination of Human Semen and Semen-Cervical Mucus Interaction, 4th ed. Cambridge, U.K.: Cambridge Univ. Press, 1999.

[9] H. Oku, M. Ishikawa, N. Ogawa, K. Shiba, and M. Yoshida, "How to track spermatozoa using high-speed visual feedback," in Proc. Int. Conf. IEEE Eng. Med. Biol. Soc., 2008, pp. 125-128.

[10] M. Berezansky, H. Greenspan, D. Cohen-Or, and O. Eitan, "Segmentation and tracking of human sperm cells using spatiotemporal representation and clustering," in Proc. SPIE_Int. Soc. Opt. Eng., vol. 6512, no. 2, 2007, pp. 65122M.1-65122M.12.

[11] L. Shi, J. Nascimento, C. Chandsawangbhuwana, E. Botvinick, and M. Berns, "An automatic system to study sperm motility and energetics," Biomed. Microdevices, vol. 10, no. 4, pp. 573-583, 2008.

[12] V. R. Nafisi, M. H. Moradi, and M. H. Nasr-Esfahani, "Sperm identification using elliptic model and tail detection," in Proc. 4th World Enformatika Conf., 2005, pp. 205-207. 
[13] R. P. Amann and D. F. Katz, "Reflections on CASA after 25 years," J. Androl., vol. 25, no. 3, pp. 317-325, 2004.

[14] T. F. Chan and L. A. Vese, "Active contours without edges," IEEE Trans. Image Process., vol. 10, no. 2, pp. 266-277, Feb. 2001.

[15] D. Davies, P. Palmer, and M. Mirmehdi, "Detection and tracking of very small low contrast objects," in Proc. Br. Mach. Vis. Conf., 1998, pp. 599608.

[16] C. M. Bishop, Pattern Recognition and Machine Learning. New York: Springer-Verlag, 2006.

[17] D. M. Tsai, P. C. Lin, and C. J. Lu, "An independent component analysisbased filter design for defect detection in low-contrast surface images," Pattern Recognit., vol. 39, no. 9, pp. 1679-1694, 2006.

[18] W. H. Wang, X. Y. Liu, and Y. Sun, "Contact detection in microrobotic manipulation," Int. J. Robot. Res., vol. 26, no. 8, pp. 821-828, 2007.

[19] N. Otsu, "Threshold selection method from gray-level histograms," IEEE Trans. Syst. Man Cybern., vol. SMC-9, no. 1, pp. 62-66, Jan. 1979.

[20] L. Itti, N. Dhavale, and F. Pighin, "Realistic avatar eye and head animation using a neurobiological model of visual attention," in Proc. SPIE-Int. Soc. Opt. Eng., 2003, vol. 5200, no. 1, pp. 64-78.

[21] C. Leung, Z. Lu, N. Esfandiari, R. F. Casper, and Y. Sun, "Detection and tracking of low contrast human sperm tail in microscopic image sequences," in Proc. IEEE Int. Conf. Autom. Sci. Eng., 2010, pp. 256268.

[22] Y. Sun, S. Duthaler, and B. J. Nelson, "Autofocusing in computer microscopy: Selecting the optimal focus algorithm," Microsc. Res. Tech. vol. 65, no. 3, pp. 139-149, 2004.

[23] B. Balaban, K. Lundin, J. M. Morrell, H. Tjellstrom, B. Urman, and P. V. Holmes, "An alternative to PVP for slowing sperm prior to ICSI," Hum. Reprod., vol. 18, no. 9, 1887-1889, 2003.

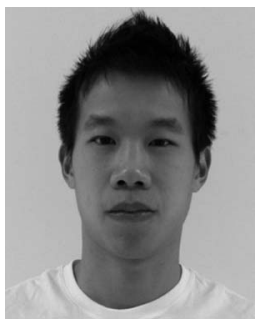

Clement Leung received the B.A.Sc. in electrical engineering from the University of British Columbia, Vancouver, BC, Canada, in 2009. He is currently working toward the Master's degree at the Electrical and Computer Engineering Department, University of Toronto, Toronto, ON.

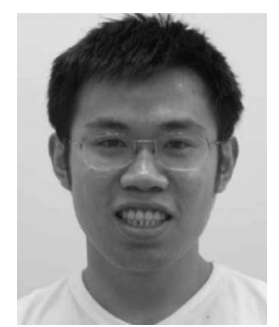

Zhe Lu (S'07-M'10) received the B.Eng. degree in control engineering from Harbin Institute of Technology, Harbin, China, in 2002, and the Ph.D. degree in mechanical engineering from the National University of Singapore, Singapore, in 2007.

$\mathrm{He}$ is currently a Postdoctoral Fellow in the Advanced Micro and Nanosystems Laboratory, Mechanical and Industrial Engineering Department, University of Toronto, Toronto, ON, Canada. His research interests include robotics, automation, and mechatronics.

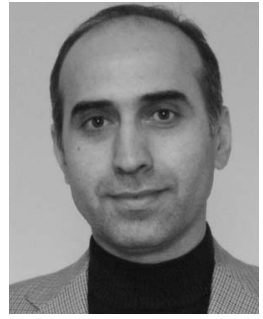

Navid Esfandiari received the D.V.M. degree from Tehran University, Tehran, Iran, in 1990, and the residency training and Ph.D. degree in medical immunology from Tehran University School of Medicine, Tehran, Iran, in 1994.

From 1994 to 2000, he was an Assistant Professor of immunology in Tehran. His fellowship was in Andrology and infertility from the Cleveland Clinic, Cleveland, OH, from 2000 to 2002 . He is currently the Director at the Assisted Reproduction Technology Laboratories, Toronto Centre for Advanced Reproductive Technology, Toronto, ON, Canada, and an Adjunct Professor in the Department of Obstetrics and Gynecology, University of Toronto, ON, Canada and the University of Tehran.

Dr. Esfandiari is a board certified HCLD by the American Board of Bioanalysis.

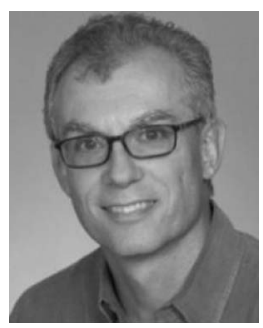

Robert F. Casper received the M.D. degree from the University of Western Ontario, London, ON, in 1973

$\mathrm{He}$ is currently a Professor at the Division of Reproductive Endocrinology and Infertility, Department of Obstetrics and Gynecology, University of Toronto, Toronto, ON, Canada, with cross appointments in the Departments of Physiology, Medicine (Division of Endocrinology), The Institute of Medical Sciences, and in the Postgraduate Department of the Faculty of Dentistry at the University of Toronto.

Dr. Casper is the Camille Dan Family Research Chair in the Translational Cell Biology and a Senior Investigator in the Samuel Lunenfeld Research Institute at Mount Sinai Hospital.

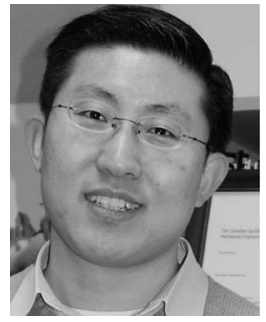

Yu Sun (S'01-M'03-SM'07) received the Ph.D. degree in mechanical engineering from the University of Minnesota, Minneapolis, in 2003.

Before joining the Faculty of Toronto in July 2004, he was a Research Scientist at the Swiss Federal Institute of Technology. He is currently an Associate Professor at the University of Toronto, Toronto, ON, Canada. His research interests include design and fabrication of microelectromechanical systems/nanoelectromechanical systems devices; micronanorobotic manipulation under optical and electron microscopes; and manipulation and characterization of biological cells, biomolecules, and nanomaterials. 